\title{
Correspondence
}

\section{The Schoch Effect to Distinguish Between Different Liquids in Closed Containers}

Nico F. Declercq, Member, IEEE, Filip Van den Abeele, Joris Degrieck, and Oswald Leroy, Senior Member, IEEE

\begin{abstract}
In different industrial branches, it is necessary to characterize liquids in closed containers. For small cans, accessibility to both sides is almost trivial. However, in industries in which larger containers are used, and especially in the dock industry, only one side is accessible practically; and damping often prevents through-transmission ultrasonic measurements or pulse echo techniques. It is known that built-in sensors can be used to determine density and wave velocity of liquids; but normally containers are not equipped with such sensors. It is also known that differences in the reflection coefficient at a solid-liquid interface can determine the density and sound velocity of liquids, but only if the difference in acoustical impedance between the solid and the liquid is small. For most containers this condition is not provided; therefore, a more sensitive method is needed. This paper reports simulations that show how identical containers, having different liquids inside, can be distinguished from one another by means of differences in the Schoch effect at a Lamb wave angle of incidence for harmonic-bounded ultrasonic beams.
\end{abstract}

\section{INTRODUCTION}

$\mathrm{T}$ HE purpose of this paper is to formulate an answer to the many requests and suggestions of several sources in the beverage industry, and especially in the dock industry, over the last couple of years for studying the possibility of fluid characterization in closed containers in a nondestructive way. In the dock industry and for port officials [1], [2], it is desirable to possess practical tools to characterize the fluid contents of containers. This can be done by removing liquid and performing chemical tests. Even though this is the only test that produces $100 \%$ certainty, it is also helpful to use other means that are equivalent to nondestructive testing. This might be due to the risk of poisoning, the lack of speed when sampling, the risk of contamination, etc.

It has been shown before that, in addition to other techniques [3], it is possible to apply ultrasound for that purpose. One method is based on time-of-flight measurements

Manuscript received April 22, 2004; accepted May 25, 2004. Research funded by a Ph.D. grant of the Institute for the Promotion of Innovation through Science and Technology in Flanders (IWTVlaanderen).

N. F. Declercq, F. Van den Abeele, and J. Degrieck are with the Soete Laboratory, Department of Mechanical Construction and Production, Ghent University, B-9000 Ghent, Belgium (e-mail: NicoF.Declercq@UGent.be).

O. Leroy is with the Interdisciplinary Research Center, Katholieke Universiteit Leuven Campus Kortrijk, B-8500 Kortrijk, Belgium.
[4]-[6]. This method requires access to both sides of a container, or at least requires containers that are small enough so that damping does not prevent sound from traversing the container. Hence, even though this method seems to work for small containers within a production line, application to large containers is not realistic.

Another method is based on laser excitation of sound in fluids [7]. This method is interesting, but the fact that it can be used only in the case of open containers makes it less applicable.

Yet another - very sensitive - method, developed by Greenwood et al. [8]-[11], applies a sensor that can be submerged in the liquid or built in the container skin; and it is actually based on the sensitivity of the reflection coefficient at the solid-liquid interface between the sensor and the liquid. Nevertheless, submerging the sensor in the liquid requires opening the container or embedding the sensor in the cargo container's skin, which is not practicable.

Ultimately, application of the sensor's principle to an existing container skin without built-in sensor is not realistic because the difference in acoustical impedance between the skin and the contained liquid is too large to make the technique sensitive enough [8]-[11]. Hence, the method developed by Greenwood et al. is probably only really practicable for pipelines [10].

The method studied here is based on beam deformations in reflection on the existing skin of a closed container, which solely requires access from one side, does not require an echo that has traversed the liquid; therefore, it is an inviting technique for relatively large containers. Because the method makes use of the existing container skin, it does not require built-in sensors. This is possible only because it is more sensitive than the reflection coefficient as used by Greenwood et al. [8]-[11]. The beam deformations are induced by the generation of Lamb waves in the container skin, and their characteristics are influenced by the properties of the liquid. It is known that harmonicbounded beams show nonspecular reflection phenomena when incident at the Rayleigh angle for liquid-solid structures [12]-[20] or at a Lamb wave generating angle on a liquid-solid-liquid structure [21]-[26]: this is called the Schoch effect.

However, all papers dealing with this effect study the situation in which a plate separates two identical liquids instead of two different ones.

In this paper, we consider an isotropic plate between two different liquids. A schematic is presented in Fig. 1. The upper liquid is always water, and the lower liquid can be of any kind. If a bounded beam is incident from the upper liquid on a plate in between that upper liquid and a lower liquid, the generation of leaky Lamb waves will induce deformation of the reflected beam, widely known as the Schoch effect, generating two reflected lobes instead 


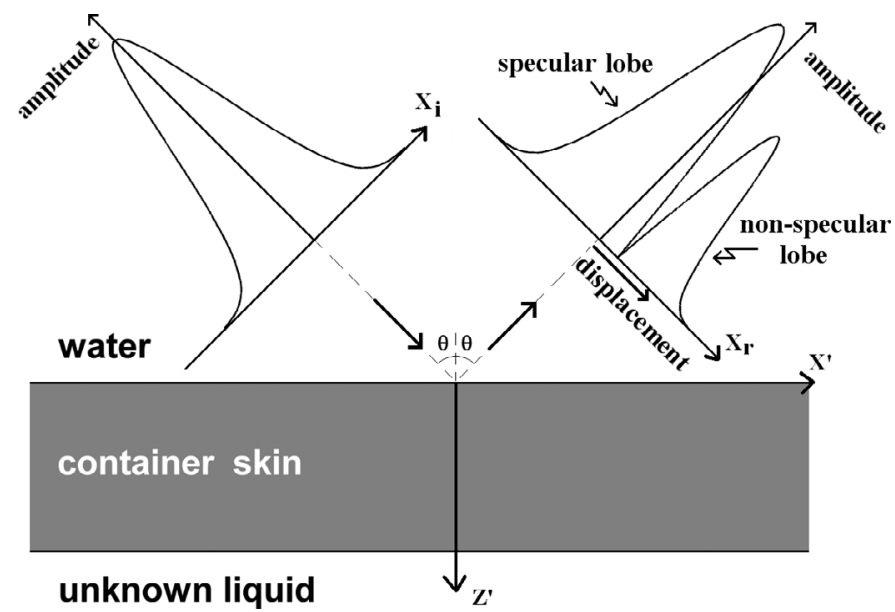

Fig. 1. Schematic of a container skin separating an unknown liquid and water. The incident and (deformed) reflected bounded-beam profiles also are shown. The short, solid arrows denote propagation direction for incident and reflected beam.

of only one. The first lobe is called the specular lobe, and its position corresponds almost perfectly with the position of a reflected beam if no deformation occurs. The second lobe is called the nonspecular lobe and is displaced along the interface. The displacement itself also is called Schoch displacement. The influence of the lower liquid on the deformed beam can be noticed in the phase and amplitude of the nonspecular lobe and in the position and depth of the null zone in between the specular and nonspecular lobe. The purpose of this paper is to show that it is possible to distinguish between two liquids in a similar container by studying differences in the Schoch effect.

It also is shown by means of a numerical simulation that measuring the characteristics of the reflected deformed beam enables one to distinguish between oil and water. This work might be an impetus for further study using phased-array techniques and it also may inspire other labs to try ultrasonic focused beams [27], [28]. It is likely that focal spot shifts also might be influenced by the characteristics of the unknown liquid.

\section{NumericAl ApPROACH}

We consider the system depicted in Fig. 1. A Gaussian incident bounded beam is considered with profile

$$
f(x)=\exp \left(-x^{2} / W^{2}\right)
$$

with $W$ the Gaussian half width. The beam itself is decomposed into infinite homogeneous plane waves by means of the Fourier transform. The sound field generated by each incident infinite homogeneous plane waves is calculated by application of the Helmholtz decomposition [29] of the particle displacement field, by incorporating the dispersion relation for bulk waves and the classical Snell's law [30] and by considering continuity of normal stress and normal displacement along each of the interfaces [31].

\section{NumericAl Results}

It can be found in Greenwood et al. [8]-[11] that, if the reflection coefficient is used to determine liquid characteristics, then the difference in acoustical impedance between the solid and the liquid must not be too large. Nevertheless, for containers, the impedance of the skin mostly differs very much from that of the contained liquid. Hence, it is not reasonable to use the reflection coefficient for liquid characterization in such containers.

In order to show this, we have calculated the reflected beam amplitude and phase at the center of the beam, for normal incidence and for a number of liquids in a container. We have taken the example of a glass plate container skin of $1.86 \mathrm{~mm}$ thickness and a $3 \mathrm{MHz}$ normal incident Gaussian beam of $1.25 \mathrm{~cm}$ Gaussian half width. For glass, the density is $2500 \mathrm{~kg} / \mathrm{m}^{3}$, and the longitudinal wave velocity is $5660 \mathrm{~m} / \mathrm{s}$ and the shear wave velocity $3520 \mathrm{~m} / \mathrm{s}$. The liquid characteristics and the numerical results are listed in Table I, in which the following parameters are used:

$$
\Delta A_{L}^{r}(x)=\frac{A_{L}^{r}(x)-A_{W}^{r}(x)}{Z_{L}-Z_{W}}
$$

$A_{L}^{r}(x)$ being the $x$-dependent reflected amplitude for a given liquid underneath the plate and $Z_{L}$ the liquid's impedance:

$$
\Delta P H_{L}^{r}(x)=\frac{P H_{L}^{r}(x)-P H_{W}^{r}(x)}{Z_{L}-Z_{W}}
$$

$P H_{L}^{r}(x)$ being the $x$-dependent reflected phase for a given liquid underneath the plate. The values, for $L$ replaced by $W$, correspond to water. It is seen that the difference in reflected amplitude and phase for any given liquid, when compared with the ones for water underneath the plate, is poor.

One way to increase the sensitivity of the reflection coefficient for the properties of the unknown liquid is to apply multiple reflections, because then the resulting reflection coefficients are separated further from one another. Hence, the larger the number of contacts between the pulse and the unknown liquid, the more the pulse is influenced by the liquid. For a harmonic-incident beam, the largest contact with the liquid occurs when Lamb waves are stimulated in the container skin. The fluid will influence the characteristics of the leakage field emitted by the Lamb wave. When the Schoch effect occurs, the second (nonspecular) lobe contains most information about the liquid, for this lobe originates from the presence of a leakage field. Again, we have taken the example of a glass plate of 1.86-mm thickness and a $3 \mathrm{MHz}$ incident Gaussian beam of $1.25 \mathrm{~cm}$ Gaussian half width. From the corresponding dispersion curves of Fig. 2, it can be found that an $A_{1}$ Lamb mode will be generated at an angle of incidence of $19.36^{\circ}$. In Fig. 3 the incident beam profile together with the calculated reflected beam amplitude profile in the case of oil and in the case of water underneath the plate 
TABLE I

Liquid Characteristics and Numerical Results. ${ }^{1}$

\begin{tabular}{cccccccc}
\hline & $\rho$ & $v$ & $Z$ & & & \\
Liquid & {$\left[\mathrm{kg} / \mathrm{m}^{3}\right]$} & {$[\mathrm{m} / \mathrm{s}]$} & {$\left[\mathrm{kg} / \mathrm{m}^{2} \mathrm{~s}\right]$} & $A_{L}^{r}(0)$ & $\frac{P H_{L}(0)}{\pi}$ & $\Delta A_{L}^{r}(0) \times 10^{9}$ & $\Delta P H_{L}^{r}(0) \times \frac{10^{5}}{\pi}$ \\
\hline Methanol & 791 & 1103 & 872473 & 0.9975 & -0.138 & -2.4690 & 3793 \\
Acetone & 791 & 1174 & 928634 & 0.9981 & -0.1091 & -3.8087 & 6609 \\
Ethanol & 790 & 1207 & 953530 & 0.9968 & -0.1008 & -1.5195 & 2574 \\
Gasoline & 803 & 1250 & 1003750 & 0.9981 & -0.136 & -4.4094 & 8913 \\
Kerosene & 810 & 1320 & 1069200 & 0.9984 & -0.1168 & -5.8422 & 13821 \\
Benzene & 870 & 1295 & 1126650 & 0.9916 & -0.0831 & 12.4522 & -35705 \\
Sunflower oil & 920 & 1450 & 1334000 & 0.9879 & -0.1635 & 55.4794 & -381122 \\
Cyclohexanole & 962 & 1450 & 1394900 & 0.9874 & -0.1644 & 101.0575 & -1189447 \\
Water & 1000 & 1480 & 1480000 & 0.996 & -0.133 & - & - \\
Nitromethane & 1130 & 1330 & 1502900 & 0.9958 & -0.1029 & -8.7336 & -374201 \\
Sea water & 1025 & 1531 & 1569275 & 0.9961 & -0.1298 & 1.1201 & 14388 \\
Glycerin & 1260 & 1904 & 2399040 & 0.9221 & 0.009 & -80.4099 & -87314 \\
Bromoform & 2890 & 920 & 2658800 & 0.9567 & -0.1827 & -33.3389 & -28142 \\
Mercury & 13500 & 1450 & 19575000 & 0.9851 & 0.0033 & -0.6023 & -24 \\
\hline
\end{tabular}

${ }^{1}$ The central reflected amplitude $A_{L}^{r}(0)$, the central reflected phase $P H_{L}(0)$, and the differences of those parameters for a given liquid underneath the plate, compared to their values in the case of water underneath the plate, $\Delta A_{L}^{r}(0)$, respectively, $\Delta P H_{L}^{r}(0)$. The density $\rho$, plane wave velocity $v$ and the impedance $Z$ of the listed liquids is also given.

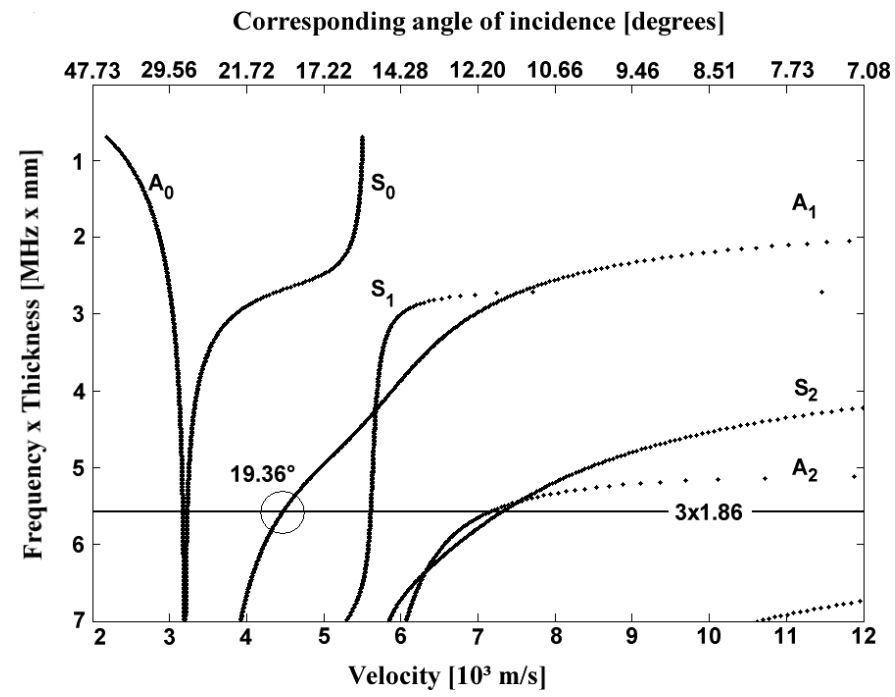

Fig. 2. Dispersion curves for the glass plate under consideration. It is seen that an angle of incidence of $19.36^{\circ}$ corresponds with the stimulation of the $A_{1}$ mode when a $3 \mathrm{MHz}$ ultrasonic beam is incident on a plate of $1.86-\mathrm{mm}$ thickness.

are shown for an angle of incidence of $19.36^{\circ}$ corresponding with stimulated $A_{1}$ Lamb waves. It is seen that the reflected profiles differ considerably. When the reflected beam phase profiles are compared, one notices a considerable difference too (Fig. 4). If we define $x^{*}$ as the position in which the nonspecular reflected lobe has maximum amplitude, then for sunflower oil $\Delta A_{L}^{r}\left(x^{*}\right)=-1144 \times 10^{-9}$ and $\Delta P H_{L}^{r}\left(x^{*}\right)=6379 \times 10^{-10} \pi$. These values are much larger than the values for normal incidence of Table I. In other words, the use of the Schoch effect is much more sensitive than the use of the reflection coefficient. However, contrary to the method of Greenwood et al. [8]-[11], when the Schoch effect is used to obtain information about the unknown liquid, it is not possible to come to a direct

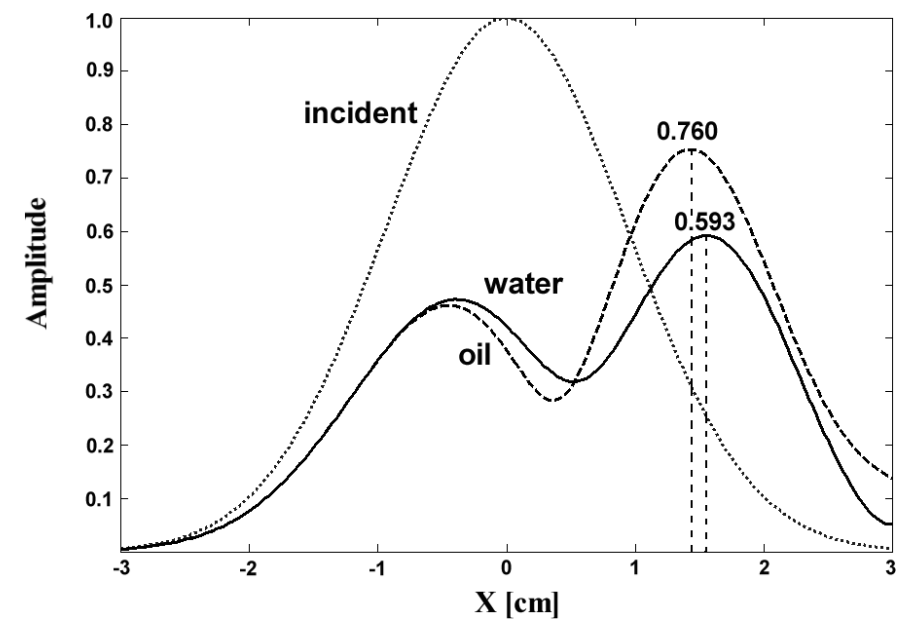

Fig. 3. Reflected amplitude profiles for a $3 \mathrm{MHz}$ bounded-beam incident at $19.36^{\circ}$ on a 1.86 -mm thick glass plate, when the unknown liquid underneath the container skin is water (solid line) or sunflower oil (dashed line). The incident-beam profile is given in a dotted line. The maximum amplitude of the nonspecular reflected beam is explicitly given.

inversion method to find the liquid characteristics from the reflected profile. When inversion is necessary, optimization procedures must be applied in order to estimate the liquid parameters. Nevertheless, it is not certain that the liquid parameters corresponding to a given reflected profile are unique. Therefore, the method presented here can be used to discriminate between given liquids (e.g., water and oil), rather than to find out the exact physical parameters of an unknown liquid.

\section{Conclusions}

An overview has been given of different techniques to characterize liquids in containers and the necessity of intro- 


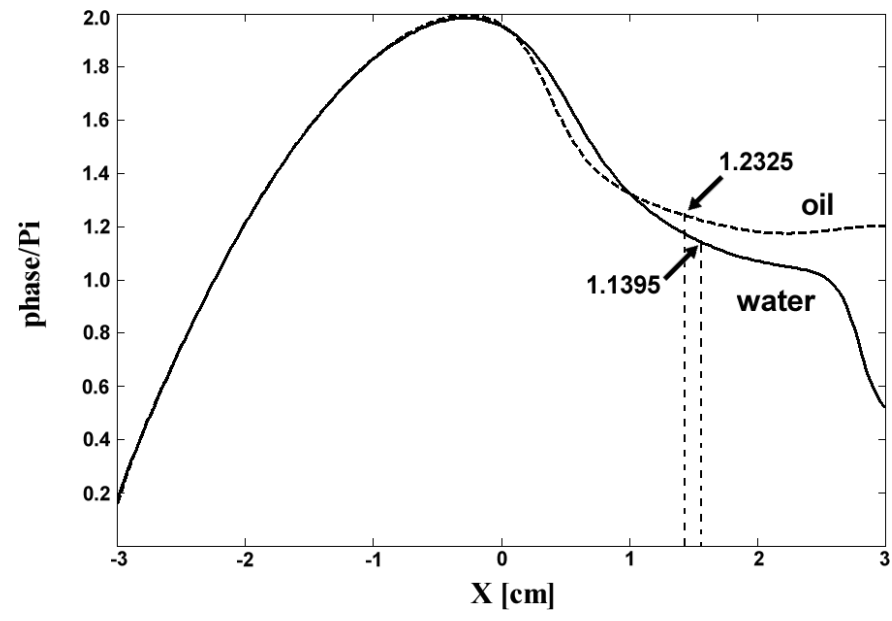

Fig. 4. Corresponding phase profiles for the geometry of Fig. 3. The phase at the position corresponding with the maximum amplitude of Fig. 3 is explicitly given.

ducing a new technique based on the Schoch effect caused by Lamb wave stimulation in the skin of a container. It is shown that the Schoch effect can be used to distinguish between different liquids in containers. The method does not apply built-in or submerged sensors and does not require opening of the container. Numerical comparison was made between the sensitivity of the outlined method and another technique that is based on the reflection coefficient. An example was given for a glass container, in which it is seen that the Schoch effect is much more sensitive than the reflection coefficient. We believe that the presented technique can be very effective, due to its flexibility and its nonrequirement of built in sensors, if it is combined with phased-array techniques to emit and receive the involved sound fields.

\section{REFERENCES}

[1] Newsletter of the Contact Group of Customs Managers of Northern Ports in the EU: Rotterdam, Antwerp, Le Havre, Felixstowe, and Hamburg, vol. 3, pp. 1-6, July 2002.

[2] Containerscan op de Rotterdamse Maasvlakte: Brochure of The Netherlands Port Customs. Den Haag, The Netherlands: External and Internal Communication, May 1999. (in Dutch)

[3] M. P. Snell, "Gamma-ray technology: The practical container inspection alternative," Port Technology Int., vol. 9, no. 3, pp. 83-91.

[4] J. Abrehouch, R. Latif, A. Moudden, and B. Faiz, "Non destructive ultrasonic characterization of canned fish oil," Acta Acustica United with Acustica, vol. 88, pp. 587-591, 2002. (in French)

[5] J. M. Doyle, "Detector can scan containers for contraband without unsealing," Aviation Weeks Homeland Security 83 Defense, February 20, 2003.

[6] "PASS (Product Acoustic Signature System)," http:// www.mehlgriffinbartek.com/html/press_frames.htm.

[7] S. Petillion, N. Van Riet, and C. Glorieux, "Laser ultrasonic characterization of complex acoustic properties of viscous liquids," presented at Ultrasonics Int., 2003, Granada, Spain.

[8] M. S. Greenwood, J. R. Skorpik, J. A. Bamberger, and R. V. Harris, "On-line ultrasonic density sensor for process control of liquids and slurries," Ultrasonics, vol. 37, pp. 159-171, 1999.

[9] M. S. Greenwood and J. A. Bamberger, "Measurement of viscosity and shear wave velocity of a liquid or slurry for on-line process control," Ultrasonics, vol. 39, pp. 623-630, 2002.
[10] — , "Ultrasonic sensor to measure the density of a liquid or slurry during pipeline transport," Ultrasonics, vol. 40, pp. 413417, 2002.

[11] M. S. Greenwood and J. C. Lail, "Ultrasonic fluid densitometry and densitometer," J. Acoust. Soc. Amer., vol. 104, no. 2, p. 614,1998

[12] M. A. Breazeale, L. Adler, and G. W. Scott, "Interaction of ultrasonic waves incident at the Rayleigh angle onto a liquidsolid interface," J. Appl. Phys., vol. 48, no. 2, pp. 530-537, 1977.

[13] M. A. Breazeale, L. Adler, and L. Flax, "Reflection of a Gaussian ultrasonic beam from a liquid-solid interface," J. Acoust. Soc. Amer., vol. 56, no. 3, pp. 866-872, 1974.

[14] T. D. K. Ngoc and W. Mayer, "Numerical integration method for reflected beam profiles near Rayleigh angle," J. Acoust. Soc. Amer., vol. 67, no. 4, pp. 1149-1152, 1980.

[15] A. B. Temsamani, S. Vandenplas, and L. Van Biesen, "Experimental investigation of bounded beam reflection from plane interfaces in the vicinity of leaky waves angles," Ultrasonics, vol. 38, pp. 749-753, 2000.

[16] M. de Billy and I. Molinero, "On the nonobservance of nonspecular bounded beam reflection effects of Lamb modes," J. Acoust. Soc. Amer., vol. 83, no. 4, pp. 1249-1254, 1988.

[17] J. Pott and J. G. Harris, "Scattering of an acoustic Gaussian beam from a fluid-solid interface," J. Acoust. Soc. Amer., vol. 76, no. 6, pp. 1829-1838, 1984.

[18] J. M. Claeys and O. Leroy, "Reflection and transmission of bounded sound beams on half-spaces and through plates," $J$. Acoust. Soc. Amer., vol. 72, no. 2, pp. 585-590, 1982

[19] T. Kundu, "On the nonspecular reflection of bounded acoustics beams," J. Acoust. Soc. Amer., vol. 83, no. 1, pp. 18-24, 1988.

[20] W. G. Neubauer, "Ultrasonic reflection of a bounded beam at Rayleigh and critical angles for a plane liquid-solid interface," $J$. Appl. Phys., vol. 44, no. 1, pp. 48-55, 1973.

[21] T. D. K. Ngoc and W. G. Mayer, "Influence of plate mode structure and Gaussian beam profile characteristics on ultrasonic reflection and transmission," IEEE Trans. Sonics Ultrason., vol. SU-29, no. 2, pp. 112-114, 1982.

[22] T. J. Plona, L. E. Pitts, and W. G. Mayer, "Ultrasonic bounded beam reflection and transmission effects at a liquid/solidplate/liquid interface," J. Acoust. Soc. Amer., vol. 59, no. 6, pp. 1324-1328, 1976.

[23] K. W. Ng, T. D. K. Ngoc, J. A. McClure, and W. G. Mayer "Nonspecular transmission effects for ultrasonic beams incident on a solid plate in a liquid," Acustica, vol. 48, pp. 169-173, 1981.

[24] T. D. K. Ngoc and W. G. Mayer, "A general description of ultrasonic nonspecular reflection and transmission effects for layered media," IEEE Trans. Sonics Ultrason., vol. SU-27, no. 5, pp. 229-236, 1980.

[25] L. E. Pitts, T. J. Plona, and W. G. Mayer, "Theory of nonspecular reflection effects for an ultrasonic beam incident on a solid plate in a liquid," IEEE Trans. Sonics Ultrason., vol. SU-24, no. 2, pp. 101-109, 1977.

[26] S. Zeroug and L. B. Felsen, "Nonspecular reflection of two- and three-dimensional acoustic beams from fluid-immersed planelayered elastic structures," J. Acoust. Soc. Amer., vol. 95, no. 6, pp. 3075-3089, 1994.

[27] T. E. Matikas, M. Rousseau, and P. Gatignol, "Theoretical analysis for the reflection of a focussed ultrasonic beam from a fluidsolid interface," J. Acoust. Soc. Amer., vol. 93, no. 3, pp. 14071416, 1993.

[28] P. B. Nagy, K. Cho, L. Adler, and D. E. Chimenti, "Focal shift of convergent ultrasonic beams reflected from a liquid-solid interface," J. Acoust. Soc. Amer., vol. 81, no. 4, pp. 835-839, 1987.

[29] J. D. Achenbach, Wave Propagation in Elastic Solids. Amsterdam: North-Holland, 1975.

[30] N. F. Declercq, J. Degrieck, and O. Leroy, "On the generalized Snell's law and its possible relation to coherent backscattering of ultrasonic waves," Appl. Phys. Lett., vol. 84, no. 21, pp. 42454247, 2004

[31] A. H. Nayfeh, Wave Propagation in Layered Anisotropic Media with Applications to Composites. Amsterdam: North-Holland, 1995. 